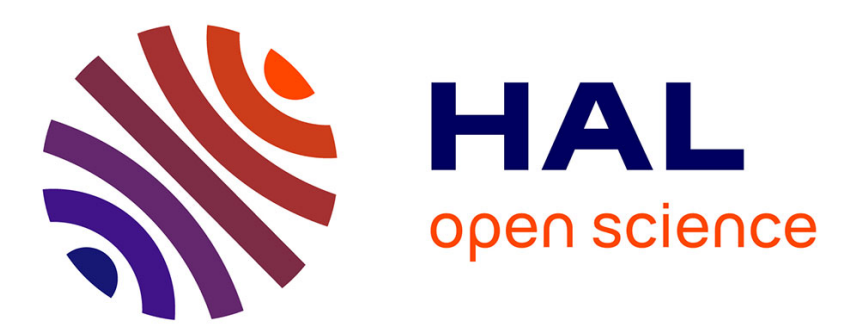

\title{
Le Louvre des femmes. Sur quelques présupposés à l'égard desfemmes dans les musées en France au XIXe siècle
}

\author{
Charlotte Foucher Zarmanian
}

\section{To cite this version:}

Charlotte Foucher Zarmanian. Le Louvre des femmes. Sur quelques présupposés à l'égard desfemmes dans les musées en France au XIXe siècle. Romantisme: la revue du dix-neuvième siècle, 2016. hal-03020194

\author{
HAL Id: hal-03020194 \\ https://hal.science/hal-03020194
}

Submitted on 23 Nov 2020

HAL is a multi-disciplinary open access archive for the deposit and dissemination of scientific research documents, whether they are published or not. The documents may come from teaching and research institutions in France or abroad, or from public or private research centers.
L'archive ouverte pluridisciplinaire HAL, est destinée au dépôt et à la diffusion de documents scientifiques de niveau recherche, publiés ou non, émanant des établissements d'enseignement et de recherche français ou étrangers, des laboratoires publics ou privés. 


\title{
LE LOUVRE DES FEMMES. SUR QUELQUES PRÉSUPPOSÉS À L'ÉGARD DES FEMMES DANS LES MUSÉES EN FRANCE AU XIX ${ }^{E}$ SIĖCLE
}

\author{
Charlotte Foucher Zarmanian
}

Armand Colin | « Romantisme »

2016/3 n 173 | pages 56 à 67

ISSN 0048-8593

ISBN 9782200930790

Article disponible en ligne à l'adresse :

https://www.cairn.info/revue-romantisme-2016-3-page-56.htm

Distribution électronique Cairn.info pour Armand Colin.

(C) Armand Colin. Tous droits réservés pour tous pays.

La reproduction ou représentation de cet article, notamment par photocopie, n'est autorisée que dans les limites des conditions générales d'utilisation du site ou, le cas échéant, des conditions générales de la licence souscrite par votre établissement. Toute autre reproduction ou représentation, en tout ou partie, sous quelque forme et de quelque manière que ce soit, est interdite sauf accord préalable et écrit de l'éditeur, en dehors des cas prévus par la législation en vigueur en France. Il est précisé que son stockage dans une base de données est également interdit. 


\section{Le Louvre des femmes. Sur quelques présupposés à l'égard des femmes dans les musées en France au XIX $X^{e}$ siècle}

En 2005, Woody Allen situe l'une des scènes de son film Match Point dans un musée : la Tate Modern de Londres. Dans cette scène de basculement, cruciale pour la suite du film et la destinée de ses héros, Chris retrouve par hasard au musée, Nola, une jeune actrice dont il a été follement épris mais qui, selon ses conceptions, n'était pas de classe sociale assez élevée pour qu'il puisse l'envisager comme épouse et mère de ses enfants. Le choix du musée comme lieu de retrouvailles n'est pas fortuit dans ce film qui raconte l'ascension sociale d'un "Rastignac " $\mathrm{du} \mathrm{XXI}{ }^{\mathrm{e}}$ siècle prêt à tout pour se faire une place dans la haute bourgeoisie londonienne. Il reconduit en effet plusieurs topoï associés au musée et à ses représentations qui ont précisément cours au XIX ${ }^{\mathrm{e}}$ siècle, période fondatrice pour son histoire et son institutionnalisation. S’il mobilise le musée comme un lieu social signant l'appartenance aux classes élevées, parce que jugées cultivées, de la société ${ }^{1}$, il le réactive aussi comme un espace du désir. Labyrinthique, le musée est utilisé comme révélateur psychologique de ses personnages. D'abord filtrée par les vitres et les reflets qui s'y impriment, la jeune femme convoitée, Nola, est fuyante, trouble. Dans la scène suivante, elle se détache plus nettement devant la surface peinte d'un tableau abstrait, jusqu'à ce que le cadrage l'enferme définitivement dans/sur cette œuvre. Nola devient là l'incarnation d'un fantasme qui ne pourra désormais plus échapper à la ténacité de Chris, lequel œuvrera jusqu’à la fin du film pour la conserver dans cet état fétichisé.

$\mathrm{Au} \mathrm{XIX}^{\mathrm{e}}$ siècle, le Grand Dictionnaire universel revient sur l'étymologie du terme " musée ». Provenant d'abord du grec mouseion, signifiant littéralement "temple des muses ", il devient en latin museum, c'est-à-dire "cabinet d'hommes de lettres ${ }^{2}$ ". Intéressante en termes de genres, cette évolution sémantique atteste du passage d'un territoire féminin à un territoire connoté au masculin, affirmant une différenciation sexuée que Chantal Georgel confirme dans le chapitre introductif de La Jeunesse des musées intitulé, de manière éloquente, "Premiers muséums, premiers hommes :

1. Voir Dominique Poulot, «L'invention de la bonne volonté culturelle : l'image du musée au $\mathrm{XIX}^{\mathrm{e}}$ siècle », Le Mouvement social, $\mathrm{n}^{\circ}$ 131, avril-juin 1985, p. 45-46.

2. Voir Pierre Larousse (dir.), Grand Dictionnaire universel du XIX siècle, t. XI, Genève - Paris, Slatkine, 1982, p. 716-717. 
la formation initiale des collections ${ }^{3} »$. L'historienne d'art y examine les cabinets de curiosités et les collections de personnalités - tous hommes sans exception soucieuses de construire un patrimoine personnel, avant l'apparition à proprement parler des musées, c'est-à-dire « des espaces réunissant des œuvres d'art, des objets de curiosité voire des produits industriels appartenant à l'État, à un département ou à une commune et exposés dans un édifice public ${ }^{4}$ ».

Certes, la prédominance du masculin est réelle dans la conception, la direction et la perpétuation des musées au XIX siècle, mais les femmes n'en sont pas totalement exclues. On songe ainsi aux visiteuses, salonnières, copistes, sujets d'œuvres d'art ou encore collectionneuses, nombreuses à faire vivre ces lieux patrimoniaux. Pourtant, peu d'études leur ont été consacrées. Sans avoir la prétention de combler l'ensemble de ce manque, cet article souhaite examiner un certain nombre de clichés véhiculés sur elles, en prenant pour cadre topographique et temporel le Louvre et le XIX ${ }^{e}$ siècle qui a justement vu leur conquête progressive d'un espace public ${ }^{5}$. L'étude des écarts entre les présupposés et les pratiques réelles permettra d'interroger la flânerie, la copie et la consommation comme autant de concepts actifs pouvant engager des interactions entre les musées et les femmes, pour poser les jalons d'une autre histoire des musées au prisme du genre.

\section{FLÂNEUSES}

Au XIX ${ }^{e}$ siècle, le musée est d'abord perçu comme un écrin idéal pour les rencontres amoureuses dans lequel la femme s'assimile aisément à la séductrice. Le onzième chapitre du roman Mensonges de Paul Bourget dépeint, par exemple, le rendez-vous que Suzanne donne au poète René dans le Salon carré du Louvre ${ }^{6}$. L’héroïne met ici à profit les ressources du lieu pour parvenir à séduire le jeune poète : une accessibilité propice faite d'escaliers et de couloirs, un parquet glissant permettant de se rapprocher de l'amant convoité, ainsi qu'un salon isolé et silencieux plongé dans une demi-clarté

3. Voir Chantal Georgel (dir.), « Premiers muséums, premiers hommes : la formation initiale des collections », La Jeunesse des musées. Les musées de France au XIX siècle, musée d'Orsay, 7 février 8 mai 1994, Paris, RMN, 1994, p. 19-35.

4. La Grande Encyclopédie. Inventaire raisonné des sciences, des lettres et des arts, t. XXIV, Paris, Société anonyme de la Grande Encyclopédie, [s. d.], p. 591.

5. Le rapport de la femme à l'espace public est plus que complexe, car même si, selon le principe de séparation des sphères, la sphère privée est assignée à la femme, la sphère publique ne lui est pas totalement fermée. En 1990, Janet Wolff explique que le «flâneur », proposé par Charles Baudelaire comme celui qui se balade dans la ville en observant et en étant observé, est impensable au féminin. Selon Wolff, la femme - et tout particulièrement la femme bourgeoise ou appartenant aux classes moyennes de la société - qui se promènerait seule, sans la compagnie de son époux, serait rapidement suspectée d'être une prostituée, une veuve, une femme âgée, une lesbienne ou encore une vagabonde, c'est-à-dire autant de figures considérées à l'époque comme marginales et souvent exclues du système social. Cet essai a provoqué un vif débat touchant à la place des femmes dans les territoires géographiques de la modernité. Voir Janet Wolff, « The Invisible Flâneuse. Women and Literature of Modernity », Feminine Sentences. Essays on Women and Culture, Cambridge, Polity Press, 1990, p. 34-50 ; Elizabeth Wilson, « The Invisible Flâneur », The Contradictions of Culture. Cities, Culture, Women, Londres, SAGE, 2001, p. 72-89 ; Nancy Forgione, «Everyday Life in Motion: The Art of Walking in Late Nineteenth-Century Paris », The Art Bulletin, vol. 87, $\mathrm{n}^{\circ}$ 4, décembre 2005, p. 674-675.

6. Voir Paul Bourget, « Déclarations », Mensonges, Paris, Alphonse Lemerre, 1890 [1887], p. 237-258. 
et composé d'un confortable divan sur lequel le couple poursuivra l'échange. Le musée devient aussi le cadre idéal pour manifester le caractère trompeur de son héroïne. Devant les œuvres, Suzanne sait trouver les poses adéquates et se glisser harmonieusement dans ce lieu esthétique tout à fait propice à l'embellir. Tel un tableau vivant, elle reprend, là, les atours des modèles féminins des œuvres d'art, jusqu'à susciter l'intérêt de son interlocuteur et donner le goût de l'ekphrasis au narrateur :

Suzanne, heureuse, jusqu'au ravissement, de cette promenade parmi les toiles qu'elle faisait semblant de regarder, s'amusait avec délices de l'impression que sa beauté produisait sur son compagnon [...] [Lui,] regardait Suzanne marcher, sœur vivante des beautés aristocratiques évoquées sur les toiles par les maîtres anciens. Ses cheveux dorés brillaient sous le chapeau du matin. Son buste se moulait dans une espèce de courte jaquette en astrakan. La petite étoffe grise de sa jupe tombait en plis souples. Elle tenait à la main un manchon, assorti à son corsage, d'où échappait un coin de mouchoir brodé, et elle élevait par instants ce petit manchon au-dessus de ses yeux, afin de se ménager le jour nécessaire à bien voir le tableau. [...] Elle qui n'aurait pas su distinguer un Rembrandt d'un Pérugin, ou un Ribera d'un Watteau, tant son ignorance était absolue, elle avait une façon d'écouter ce que lui disait René, et un art d'abonder dans le sens de ses idées, qui aurait fait illusion à de plus habiles connaisseurs du mensonge féminin, que ce poète de vingt-cing ans ${ }^{7}$.

Le vocabulaire employé illustre une puissance visuelle de la femme inversement proportionnelle à sa puissance intellectuelle. Le chapitre souligne en effet la superficialité et l'amateurisme de son héroïne, au sein d'un strict partage des rôles où cette dernière est l'objet du voir quand l'homme, lui, détient le savoir.

Dans un essai, Isabelle Baudino a pourtant prouvé que les femmes étaient bien loin d'être hermétiques à l'art et ignorantes ${ }^{8}$. Focalisant son attention sur les voyageuses britanniques en villégiature à Paris au début du $\mathrm{XIX}^{\mathrm{e}}$ siècle et pour lesquelles le Louvre forme un passage obligé, elle analyse plusieurs récits viatiques écrits par les femmes pendant ce périple initiatique et éducatif du Grand Tour. Elle évoque notamment la soif de connaissance d'Anne Plumptre dont les visites régulières pendant huit mois ne lui ont pas permis de se familiariser autant qu'elle l'aurait souhaité avec les collections, ou encore le degré d'instruction de Marianne Colston qui cite une longue liste de maîtres anciens visibles au musée mais qui regrette de ne pas trouver les tableaux de Raphaël pourtant repérés dans le catalogue ${ }^{9}$. Ces récits révèlent que les femmes peuvent ainsi utiliser la visite au musée comme un levier d'émancipation

7. Ibid., p. 246-247 (termes mis en gras par moi-même).

8. Isabelle Baudino, «Les voyageuses britanniques à Paris : un point de vue féminin sur l'art? », Plumes et pinceaux. Discours de femmes sur l'art en Europe (1750-1850), Mechthild Fend, Melissa Hyde, Anne Lafont (dir.), vol. I, Dijon - Paris, Les presses du réel - INHA, 2012, p. 153-184.

9. Marianne Colston, Journal of a Tour in France, Switzerland, and Italy. During the Years 1819, 20 , and 21, Paris, Galignani, 1822, cité dans : Plumes et pinceaux. Discours de femmes sur l'art en Europe (1750-1850) - Anthologie, Anne Lafont (éd.), vol. II, Dijon - Paris, Les presses du réel - INHA, 2012, p. 206-213. 
culturelle et sociale. Démontrant qu'elles sont capables de maîtriser des références érudites et de porter un jugement personnel sur les œuvres, elles s'affirment dans une culture du connoisseurship connotée au masculin comme des "femmes de goût ${ }^{10}$ ". En même temps, pour ne pas perturber les attentes et les convenances, elles n'étalent pas ostensiblement leurs connaissances et, souvent, prennent le soin de distiller leur opinion avec parcimonie et modestie. Le Guide des dames au musée royal de peinture paru en 1830 est à ce sujet un bel exemple de cet agency mobilisé par les femmes [fig. 1].

Dans l'introduction à ce livre à vocation pédagogique, adressé en premier lieu au public spécifique des jeunes filles, son auteur, la comtesse de $\mathrm{M}^{* * *}$, performe une infériorité intellectuelle qui affecte son ouvrage comme n'étant "d'aucun mérite ", ayant "l'avantage d'être à la portée de tous [et qui] n'effraiera personne ${ }^{11}$ ". La comtesse le publie d'ailleurs anonymement, défend le caractère exogène de ses connaissances et valorise le processus laborieux dont ce travail est le résultat. En même temps, elle justifie aussi son contenu par l'entraînement de son œil et se réfere par là à une certaine pratique des connaisseurs qui ont bâti leur expertise sur l'exercice visuel pour authentifier et juger de manière critique les œuvres d'art :

J'ai cherché à connaître les qualités et les défauts des grands maîtres dont nous possédons les chefs-d'œuvre ; j’ai été les étudier, former mon jugement d'après eux ; aidée des lumières de quelques savants, j'ai écrit leurs arrêts ; dans l'exercice répété et l'habitude de voir, j'ai trouvé d'utiles secours pour acquérir les connaissances qui, par suite, m’ont fait apprécier le mérite de nos artistes; par ce moyen, j’ai fixé mon jugement quant à la science ${ }^{12}$.

L'auteur fait preuve d'un réel discernement, prenant systématiquement parti pour juger les œuvres. Elle sélectionne les artistes qu'elle juge les meilleurs, élabore une véritable histoire de l'art par école et prend position sur l'iconographie, la composition et le style de l'œuvre. Par souci pédagogique, elle construit aussi, en fin d'ouvrage, un lexique spécifique du vocabulaire technique employé - méplat, clair-obscur, perspective aérienne, ordonnance y sont entre autres décrits avec précision. Le jeu sur la minimisation de son expertise ne prouve en aucun cas son ignorance et son incompétence pour parler d'art.

\section{COPISTES}

Dans les musées, les femmes ne sont pas uniquement spectatrices des œuvres, elles en conçoivent aussi. Le Louvre incarne, dans cette perspective, non seulement un lieu de contemplation et d'observation mais également un lieu de formation. Et comme les visiteuses, les femmes artistes doivent affronter la critique qui les assimile

10. Voir Ann Bermingham, « The Aesthetics of Ignorance: The Accomplished Woman in the Culture of Connoisseurship », The Oxford Art Journal, $16: 2$, 1993, p. 3-20.

11. Voir Madame la Comtesse de $\mathrm{M}^{* * *}$, Guide des dames au musée royal de peinture, Paris, Chez Renard, 1830 , p. 6.

12. Ibid., p. 6-7. 


\section{GUIDE}
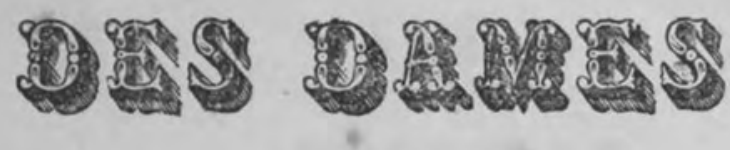

A $\mathbf{U}$

\section{MUSÉE ROYAL DE PEINTURE.}

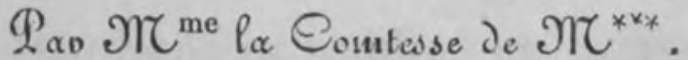
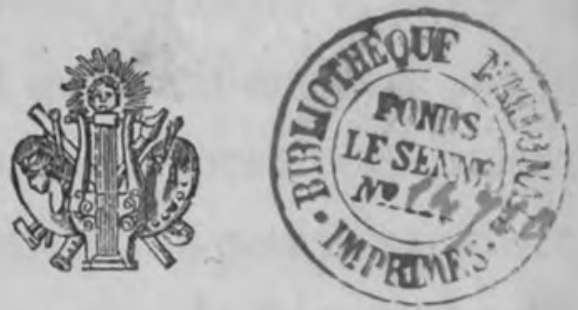

PARIS,

A LA LIBRAIRIE DU COMMERCE,

\section{CHEZ RENARD, LIBRAIRE,}

RUE SAINTE-ANNE, $\mathrm{N}^{\circ} 7 \mathrm{I}$.

\section{0}

Figure 1. Madame la Comtesse de $\mathrm{M}^{* * *}$, Guide des dames au musée royal de peinture, Paris, Chez Renard, 1830, @droits réservés. 
le plus souvent à des barbouilleuses envahissant le musée. L'ouvrage Les Pensionnaires $d u$ Louvre du critique d'art Louis Leroy est certainement le plus prolixe à rassembler la multitude des stéréotypes circulant à l'époque sur elles [fig. 2] ${ }^{13}$. Prolongeant les nombreuses physiologies illustrées représentatives de l'engouement du temps pour la satire sociale, cet ouvrage pointe les ridicules et les travers des copistes au travers d'une conversation menée par Leroy et un dénommé Potet, rapin habitué des lieux. Plusieurs archétypes sont dépeints et mis en scène dans des dialogues misogynes : la ménopausée "à la tête de boule-dogue " côtoie la mélancolique faisant des copies pour vivre, la frivole n'existant que dans le regard et l'ombre du masculin, ou encore la badigeonneuse " cuisinant sa peinture comme un simple miroton ${ }^{14}$ ".

Confrontant cette perception faussée à des sources précises mêlant archives, correspondances et témoignages, Paul Duro et Séverine Sofio se sont attelés à complexifier cette population pas aussi féminisée et incompétente que le discours ambiant - de surcroît satirique - le prétendait alors. Paul Duro a ainsi expliqué que, sous le Second Empire, l'État créa la catégorie professionnelle des peintres copistes pour fonder un art officiel valorisant son image et sa puissance. Il prit l'habitude de commander à des artistes, femmes et hommes confondus, des portraits dynastiques ou des œuvres destinées à des lieux publics ${ }^{15}$. Dans sa thèse, Séverine Sofio a aussi prouvé que la copie était loin d'être un domaine réservé aux femmes ou aux artistes " ratés », mais qu'elle constituait en réalité un passage obligé dans toute formation professionnelle et une ressource économique nécessaire pour la production d'une œuvre personnelle ${ }^{16}$. Grâce à la copie, une femme avait facilement accès à la peinture religieuse et pouvait donc, de manière contournée, démontrer ses talents dans le respectable genre de la peinture d'histoire ${ }^{17}$. De nombreuses sources révèlent aussi que l'une des vocations du musée, par le nombre et la variété des œuvres qu'il conserve, est de fournir à l'artiste matière à son travail et de permettre sa progression. En ce sens, le Louvre représente autant un lieu de légitimation des arts qu'un lieu de légitimité pour les artistes professionnel-les, et tout particulièrement pour les femmes qui doivent tout au long du XIX ${ }^{\mathrm{e}}$ siècle composer avec une offre pédagogique restreinte ${ }^{18}$. Lieu de sociabilité et d'émulation, le Louvre est aussi régi par de fortes conditions de promiscuité et de rivalité qui ne facilitent pas toujours son accès aux femmes, contraintes pour s'y rendre d'être chaperonnées ${ }^{19}$. La bienséance du lieu est d'ailleurs

13. Voir Louis Leroy, Les Pensionnaires du Louvre, Paris - Londres, Librairie de l'art, 1880.

14. Ibid., p. $10 ; 15$.

15. Voir Paul Duro, « Copyists in the Louvre during the Middle Decades of the Nineteenth Century », Gazette des beaux-arts, avril 1988, p. 249-254.

16. Voir Séverine Sofio, «Les copistes: "petites mains” et exposantes consacrées », "L'Art ne s'apprend pas aux dépens des mœurs! ». Construction du champ de l'art, genre et professionnalisation des artistes (1789-1848), thèse de sociologie, EHESS, 2009, p. 616-655.

17. Ibid., p. 627.

18. Alors que l'École des Beaux-arts n'ouvre officiellement aux femmes qu'en 1897 et que ces dernières doivent se tourner vers des ateliers et académies privés au coût souvent élevé, le Louvre peut apparaître comme la seule « école » gratuite, ouverte à toutes et à tous. Cet élément émane aussi d'une tradition plus historique : rappelons qu'Élisabeth Vigée-Lebrun investit les espaces du Louvre pour ses ateliers de femmes très fréquentés.

19. Voir Séverine Sofio, ouvr. cité, p. 644-654. 


\title{
LES PENSIONNAIRES DU LOUVRE
}

\author{
(CLASSE DES DAMES '
}

II

Pour ma seconde visite au Louvre, j'avais donné rendez-vous à mon cicerone Jean Potet devant les Sabines de David. Je n'y trouvai qu'une forte dame qui, le poing sur la hanche, jetait un regard satisfait sur une immense copie de ce tableau.

Un mouvement un peu brusque lui fit tomber sa brosse des mains. Elle se baissa pour la ramasser; mais, bien que de petite taille, elle ne put y arriver, le développement des muscles abdominaux et la solidité des ferrures du corset rendant la flexion sinon impossible, au moins très laborieuse.
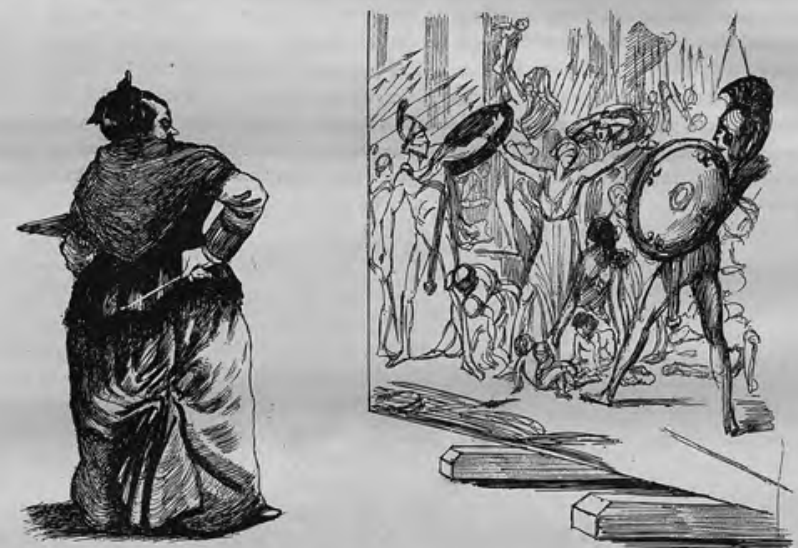

Je me précipitai pour lui en épargner la peine. Elle me remercia, et nous nous mimes à causer.

- Quelle magnifique chose! dit-elle, indiquant le tableau du maitre. Est-ce assez balancé, pondéré!

- En effet, la pondération ne laisse rien à désirer; les groupes sont réglés... mathématiquement.

- Il y a pourtant des niais qui reprochent à ses figures de poser, de manquer de mouvement.

- On a bien nié celui de la terre.

- C'est ma foi, vrai! Ah! Louis David, quel génie!

- Dans ses portraits, dans le Marat, dans les bonshommes qu'il a vus, dit une voix

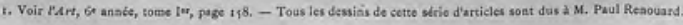

Figure 2. Louis Leroy, Les Pensionnaires du Louvre, Paris - Londres, Librairie de l'art, 1880, @droits réservés.

pointée du doigt par Gustave Flaubert dans son célèbre aphorisme : « Du Louvre : à éviter pour les jeunes filles ${ }^{20}$ " ou encore dans le portrait du journaliste anglais James Grant, incriminant sa scénographie dans l'éveil d'une soi-disant perversion féminine?

The collection of these is very large, and very valuable. But many of the figures, however admirable as works of art, are altogether unfit for female inspection.

20. Gustave Flaubert, Le Dictionnaire des idées reçues, Paris, Glyphe, 2011 [Paris, E.-L. Ferrière, 1913], p. 134. 
And yet the parts of the Louvre in which they are exhibited, are crowded with lady visitors. How ladies can deliberately walk through these apartements in the presence of gentlemen, and carefully inspect the various figures which there invite attention, appears to me surprising. The effects must be to blunt, in a greater or less degree, those delicate sensibilities of the mind which constitute the great charm of female character. I am convinced that the inferiority of the French ladies to the ladies of England in the attribute of modesty, is as much to be ascribed to the prevalence among them of paintings and statues without any drapery $[\ldots]^{21}$

Prescrivant d'une certaine façon un parcours qui leur serait spécifiquement réservé, cette appréciation révèle surtout une angoisse vis-à-vis d'une émancipation sociale des femmes qui coïnciderait avec l'expression de plus en plus affirmée de leur désir. Le tabou du regard féminin sur la nudité masculine est d'ailleurs au XIX ${ }^{\mathrm{e}}$ siècle l'une des raisons fréquemment invoquée pour interdire l'accès des femmes à l'École des beaux-arts. Contrairement à leurs collègues hommes qui peuvent travailler d'après un modèle masculin ou féminin entièrement nu, les femmes, elles, doivent habiller leurs modèles hommes d'un pagne ou d'un caleçon ; un symbole fort qui en dit long sur la persistance des inégalités dans les enseignements dispensés respectivement aux deux sexes ${ }^{22}$.

\section{BIBELOTEUSES}

Un autre cliché relatif à la visite au musée repose sur l'intérêt mercantile et décoratif de la femme pour les objets d'art. Au sein d'une époque marquée par l'essor de la consommation de masse, la bourgeoise incarnerait une ambassadrice de la vulgarité et du mauvais goût, essayant de s'approprier en vain une culture aristocratique ${ }^{23}$. Ainsi, dans le vaudeville John Bull au Louvre, Madame Florange intervient sous les traits de l'épouse d'un homme riche se baladant à l'exposition des objets de l'industrie au Louvre, et est décrite comme " prenant tout sans marchander et ne répondant aux observations de son époux que par ce mot : payez, M. le baron ${ }^{24}$ ! ". De la même façon que Jonathan Crary note un glissement des archétypes où « [l]'observateur peut par exemple s'identifier au flâneur et au consommateur en se déplaçant dans une succession infinie d'images aussi illusoires que s'il s'agissait de marchandises ${ }^{25}$ ", Neil Harris a examiné les convergences entre les musées et les grands magasins. L'historien note que ces deux espaces ont en commun d'être ouverts au public et sont construits, l'un comme l'autre, sur le mode de l'émulation. Ils reposent sur un mimétisme des

21. James Grant, Paris and its People, t. II, Londres, Saunders \& Otley, 1844, p. 198-199.

22. Voir Tamar Garb, «The Forbiden Gaze : Women Artists and the Male Nude in Late NineteenthCentury France », The Body Imaged. The Human Form and Visual Culture Since the Renaissance, Kathleen Adler, Marcia Pointon (éd.), Cambridge, Cambridge University Press, 1993, p. 33-42.

23. Voir Lisa Tiersten, "The "Vulgar Mentality" of the Bourgeois Woman Consumer », Marianne in the Market. Envisioning Consumer Society in Fin-de-siècle France, Berkeley, University of California Press, 2001, p. 76-80.

24. Voir Theaulon, Saint-Laurent, Bayard, John Bull au Louvre. Vaudeville en trois tableaux, Paris, Quoi, 1827, p. 15.

25. Jonathan Crary, L'Art de l'observateur. Vision et modernité au XIX $X^{e}$ siècle, Paris, Jacqueline Chambon, 1994 [Cambridge, Massachussetts Institute of Technology, 1990], traduction française, p. 46. 
pratiques convergeant vers une amélioration des goûts, des valeurs et des normes de conduite, plus sporadiquement diffusés dans la société ${ }^{26}$. Tous deux sont en quelque sorte des institutions capitalistes créées pour le plaisir visuel. Ceci pourrait en partie expliquer pourquoi les femmes sont plutôt bien acceptées dans ces lieux publics, lorsqu'elles en restent bien sûr aux rôles de maîtresses de maison élégantes ${ }^{27}$. Dans $A$ Museum of One's Own, Anne Higonnet a d'ailleurs bien noté que ces modes de raillerie des femmes avaient pu être contournés habilement par elles, et que la collection d'art pouvait leur offrir une précieuse chance d'affirmer leur individualité sans pour autant perturber les convenances; décorer un intérieur étant plutôt considéré autour de 1900 comme une activité appropriée pour une femme de riche condition ${ }^{28}$. Le roman Soi de Paul Adam joue d'ailleurs subtilement sur l'équivoque régissant le musée entre espace privé et espace public. L'héroïne bourgeoise de ce récit peu connu, Marthe, qui vit dans la passion exclusive d'elle-même, a tissé autour d'elle un cocon protecteur la préservant du monde extérieur. Dans cette bulle, le Louvre fait office de refuge intimiste propice à la rêverie : de salle en salle, Marthe prend, chaque mercredi, l'habitude de s'asseoir sur des "sièges profonds en velours rouge [...] s'y affala[n]t pour un repos de ses chairs pesantes ${ }^{29}$ ".

En dehors de ces récits fictionnels, les femmes ont pourtant démontré qu'elles étaient loin d'être des spectatrices passives et superficielles. Certaines jouèrent un rôle actif dans l'enrichissement des collections. Hortense Lacroix Cornu permet, par exemple, en 1861 l'acquisition de la collection Campana et la création de l'éphémère musée Napoléon III. Participant certes de manière détournée - et très involontaire à l'enrichissement du Louvre, elle parvient à convaincre directement Napoléon III d'acheter secrètement cette collection. Elle invoque officiellement la convoitise des Anglais puis des Russes pour cet achat, mais mène en réalité un dessein plus personnel : la conquête d'un poste de directeur pour son époux Sébastien Cornu qui prend activement part au rapatriement en France de la collection, et évince pour cela Émilien de Nieuwerkerke, directeur général des musées impériaux et personnalité éminente de la vie culturelle du Second Empire ${ }^{30}$. Cet exemple d'intercession féminine, assez

26. Voir Neil Harris, « Museums, Merchandising, and Popular Taste : the Struggle for Influence », Material Culture and the Study of American Life, Ian M. G. Quimby (éd.), New York, Norton, 1978, p. 141-149.

27. Je renvoie ici à l'analyse proposée par Tony Bennett, The Birth of the Museum. History, Theory, Politics, Londres - New York, Routledge, 2002 [1995], p. 28-29. Au tournant du XVIII ${ }^{\mathrm{e}}$ au XIX ${ }^{\mathrm{e}}$ siècle, l'implication des femmes est réduite dans les sphères publiques et politiques au profit d'une forte naturalisation de la féminité aliénée à la sphère domestique et aux pratiques de domesticité : la femme doit servir, nourrir et faire grandir. $\mathrm{Au} \mathrm{XIX} \mathrm{X}^{\mathrm{e}}$ siècle, se met en place une redéfinition des rôles féminins dans la sphère culturelle, où la femme se sert justement de ce nouveau statut pour ne plus apparaître comme la salonnière dominante, mais comme une aimable maîtresse de maison cultivée.

28. Voir Anne Higonnet, A Museum of One's Own. Private Collecting, Public Gift, Pittsburgh - New York, Periscope Publishing, 2009, p. 192.

29. Paul Adam, Soi, Paris, Tresse \& Stock, 1886, p. 412.

30. Voir Gianpaolo Nadalini, « La collection Campana au musée Napoléon III et sa première dispersion dans les musées français (1862-1863) », Journal des savants, 1998, p. 183-225; Bonnie Effros, «"Elle pensait comme un homme et sentait comme une femme". Hortense Lacroix Cornu (1809-1875) and the Musée des Antiquités Nationales de Saint-Germain-en-Laye », Journal of the History of Collections, vol. $24, \mathrm{n}^{\circ} 1,2012$, p. 25-43. 
atypique il faut le reconnaître, permet toutefois, par son caractère ambitieux, de renouveler les modèles de féminité et de repenser les rapports entre les sexes.

L'autre part importante que les femmes ont pu jouer résultent des donations et legs faits au musée. Fondant sa fortune dans le négoce du champagne, JeanneAlexandrine Pommery achète ainsi Les Glaneuses de Millet pour 300000 francs, afin d'empêcher ce tableau d'intégrer les collections américaines et en fait immédiatement don au Louvre en 1890 [fig. 3]. Par ce geste généreux, elle peut autant montrer son patriotisme qu'affirmer une fortune personnelle, fragilisée à l'époque par de multiples rumeurs sur de supposées difficultés financières.

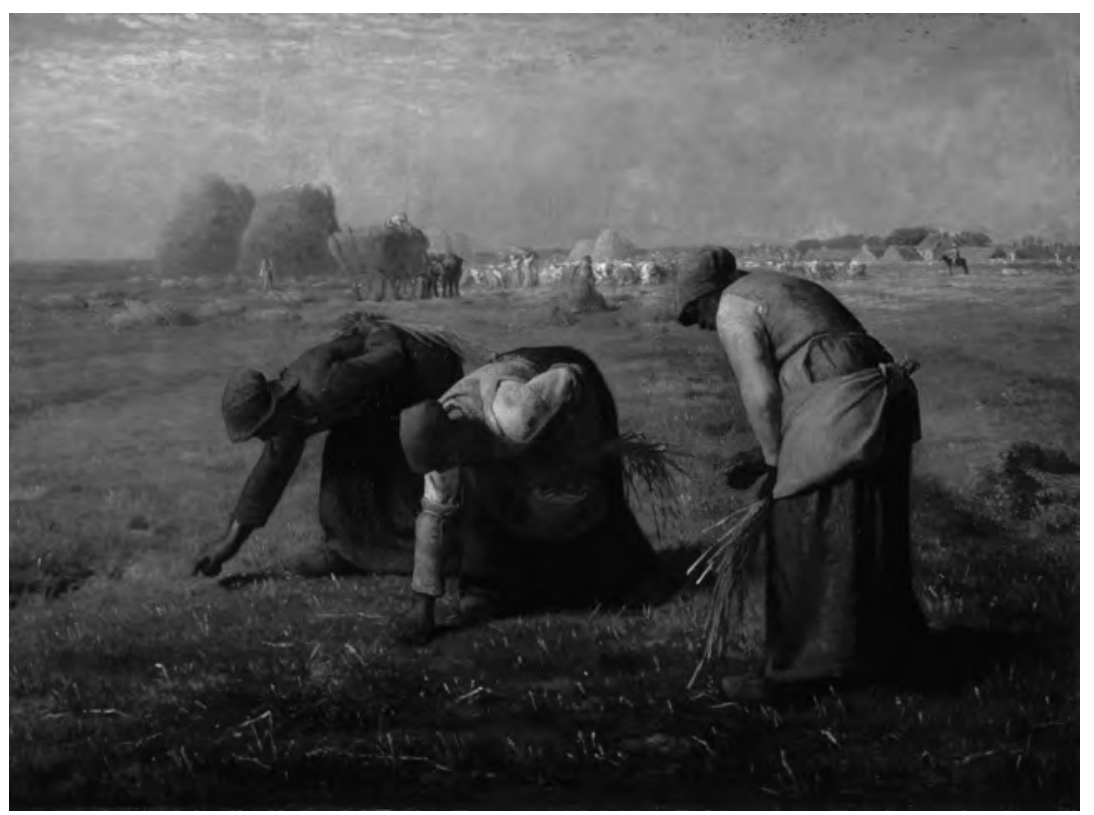

Figure 3. Jean-François Millet, Des glaneuses dit aussi Les glaneuses, 1857, huile sur toile, 83,5 x $110 \mathrm{~cm}$, Paris, musée d'Orsay, (C) Google Art Project 2.

La plupart des femmes agissent surtout au nom du couple, ce qui rend complexe la possibilité de distinguer la part de « matrimoine » dans ce " matrimonial ». D'ailleurs, des contraintes juridiques et économiques pèsent sur elles jusqu'à la fin du xix ${ }^{\mathrm{e}}$ siècle, période où elles accèdent progressivement à la propriété et peuvent disposer librement d'un héritage ${ }^{31}$. Si c'est très souvent au moment du décès d'un père ou d'un époux que les femmes donnent le patrimoine familial ou conjugal, la reconnaissance sociale du don au musée est aussi très attractive pour elles : il favorise une sortie en douceur de l'espace domestique et de l'anonymat vers la sphère publique. Citoyennes privées

31. Voir Véronique Long, Mécènes des deux mondes. Les collectionneurs donateurs du Louvre et de l'Art Institute de Chicago (1879-1940), Rennes, PUR, 2007, p. 37-38; Julie Verlaine, Femmes collectionneuses d'art et mécènes de 1880 à nos jours, Paris, Hazan, 2013, p. 28. 
de vote, ces femmes s'engagent ainsi en s'insérant dans les réseaux plus alternatifs de la philanthropie - qui s'inscrit, tout comme la mission pédagogique, dans l'histoire de l'émancipation des femmes - ou en créant de nouvelles solidarités axées sur la défense de la condition des femmes. Ainsi, l'Autoportrait d'Hortense HaudebourtLescot donné en 1867 par son élève et amie la peintre Maria Buhner, ou encore le portrait sculpté d'Anne Fortunée Léonie Garat, surintendante de la Maison de Légion d'Honneur par sa petite-fille Thérèse Clairval en 1891, montrent que la sororité est moteur dans certaines donations.

Intégrant la puissante doxa des stéréotypes assignées aux femmes dans les musées, la visite au musée, la pratique artistique et la collection d'œuvres ont été examinés ici au service d'une autre histoire des musées en France qui a très souvent compté sans les femmes ${ }^{32}$. Lieu de mixité des genres, accessible à tous, le musée au XIX ${ }^{\mathrm{e}}$ siècle n'est en réalité pas vraiment un lieu de mixité sociale. Il faudrait procéder à une étude plus large et précise de cette part féminine dans les musées - prosopographique et quantitative - pour s'apercevoir sans doute que les femmes dont on parle ne sont pas vraiment toutes les femmes. En croisant la variable du sexe avec celle de la classe sociale, on verrait rapidement qu'elles sont majoritairement parisiennes, issues de classes aisées, bourgeoises ou aristocrates. Ce présupposé persiste en ce qui concerne, plus tard, la formation des premières historiennes de l'art diplômées et conservatrices du patrimoine, très souvent sorties de l'École du Louvre, une institution souvent décrite à sa fondation, en 1882, comme un vivier de jeunes filles de bonne famille prêtes à marier. Pourtant, Louise Paschould, première femme à soutenir une thèse d'histoire de l'art en 1901, la marquise Arconati-Visconti, cofondatrice de la Société des Amis du Louvre, donnant en 1914 l'ensemble de sa collection au musée, Rachel Boyer, mécène des premiers cours d'histoire de l'art gratuits destinés à tous, Christiane Desroches-Noblecourt, l'une des premières conservatrices du patrimoine au Louvre et première enseignante à l'École du Louvre, Magdeleine Hours, directrice du laboratoire d'études scientifique de la peinture et des objets d'art au Louvre, Christiane Aulanier et Geneviève Bresc-Bautier, auteurs de travaux significatifs sur l'histoire du musée ${ }^{33}$, remettent à l'évidence en cause ces visions réductrices de superficialité, d'incompétence et de passivité. S'intégrant plutôt à une histoire des représentations, cet article pourrait, au-delà des quelques exemples évoqués, se nourrir d'une étude socio-historique plus large, soulevant des réflexions multiples relatives au rang hiérarchique des femmes dans les musées, leur mode d'insertion dans ces milieux savants et professionnels à dominante masculine ou encore leur part dans

32. Au Royaume-Uni et aux États-Unis, les études sur le sujet sont toutefois plus dynamiques, mais ne concernent en général pas les musées français. Citons juste Amy K. Levin (éd.), Gender, Sexuality and Museums, New York, Routledge, 2010.

33. Voir Christiane Aulanier, Histoire du Palais et du Musée du Louvre, Paris, Éditions des musées nationaux, 1948-1971 ; Geneviève Bresc-Bautier, Mémoires du Louvre, Paris, Gallimard - RMN, 1989 ; Le Louvre, histoire, architecture et décors, Paris, Assouline, 1995 ; Louvre : les collections du Louvre, Paris, RMN, 1999 ; Le Louvre, une histoire de palais, Paris, Musée du Louvre - Somogy, 2008. 
des domaines inhérents à la muséologie comme la conservation, la restauration, la gestion et la valorisation des collections, la médiation et le commissariat d'exposition.

(CNRS, UMR 8238, Laboratoire LEGS) 